\title{
A NEW CHARACTERIZATION OF SEPARABLE GCR-ALGEBRAS
}

\author{
TROND DIGERNES
}

\begin{abstract}
ABSTRACr. It is shown that a separable $C^{*}$-algebra $\mathfrak{A}$ is GCR if and only if the set of central projections in its enveloping von Neumann algebra $\mathfrak{B}$ is generated, as a complete Boolean algebra, by the set of open, central projections in $\mathfrak{B}$.
\end{abstract}

1. Let $\mathfrak{A}$ be a $C^{*}$-algebra, and $\mathfrak{B}$ its enveloping von Neumann algebra, that is, $\mathfrak{B}=\pi_{u}(\mathfrak{U})^{\prime \prime}$, where $\pi_{u}$ is the direct sum of all cyclic representations of $\mathfrak{A}$. The representation $\pi_{u}$ is faithful, and we may therefore consider $\mathfrak{A}$ as a sub- $C^{*}$-algebra of $\mathfrak{B}$. To each (nondegenerate) representation $\pi$ of $\mathfrak{U}$ there corresponds a projection $E^{\prime} \in \mathfrak{B}^{\prime}=\pi_{u}(\mathfrak{U})^{\prime}$ such that $\pi$ may be identified with the map $A \in \mathfrak{U} \rightarrow A E^{\prime} \in \mathfrak{B} E^{\prime}[2, \S \S 5$ and 12]. A projection $E \in \mathfrak{B}$ is said to be open if it supports a left ideal in $\mathfrak{A}$; that is, if there is a left ideal $J$ in $\mathfrak{U}$ such that $\bar{J}=\mathfrak{B} E$, where "-" denotes strong closure [1]. We let $\mathscr{P}$ denote the set of all central projections in $\mathfrak{B}, \mathscr{P}_{0}$ the set of open projections in $\mathscr{P}$ and $\left\langle\mathscr{P}_{0}\right\rangle$ the Boolean algebra generated by $\mathscr{P}_{0}$ in $\mathscr{P}$. With these notations the following has been proved by $\mathrm{H}$. Halpern and the author [5]:

1. $\mathfrak{U}$ is CCR if and only if $\mathscr{P}_{0}$ is strongly dense in $\mathscr{P}$.

2. If $\mathfrak{A}$ is GCR, then $\left\langle\mathscr{P}_{0}\right\rangle$ is strongly dense in $\mathscr{P}$.

The purpose of this paper is to obtain a converse to 2 , at least in the separable case.

For the general theory of $C^{*}$-algebras and von Neumann algebras we refer the reader to the two books of Dixmier ([2], [3]), especially $\S \S 4,5$ and 12 of [2].

2. With notations as above we have:

TheOREM. For a separable $C^{*}$-algebra $\mathfrak{A}$ the following two conditions are equivalent:

(i) $\mathfrak{A}$ is GCR;

(ii) $\left\langle\mathscr{P}_{0}\right\rangle$ is strongly dense in $\mathscr{P}$.

ProOF. (i) $\Rightarrow$ (ii). See [5].

Received by the editors April 13, 1972.

AMS 1970 subject classifications. Primary 46L05; Secondary 46L25.

Key words and phrases. $C^{*}$-algebra, enveloping von Neumann algebra, open projections, GCR-algebra.

(c) American Mathematical Society 1973 
(ii) $\Rightarrow$ (i). To prove this we use the following characterization of separable GCR algebras, due to Glimm: $\mathfrak{A}$ is GCR if and only if any two irreducible representations of $\mathfrak{A}$ with the same kernel are equivalent [4].

So let $\pi_{1}, \pi_{2}$ be irreducible representations of $\mathfrak{U}$ with $\operatorname{ker} \pi_{1}=\operatorname{ker} \pi_{2}$, and let $Q_{1}, Q_{2}$ be the central supports of the minimal projections in $\mathfrak{B}^{\prime}=\pi_{u}(\mathfrak{U})^{\prime}$ corresponding to $\pi_{1}$ and $\pi_{2}$ respectively. (The central support $C_{E}$ of a projection $E$ in a von Neumann algebra $\mathfrak{B}$ is defined by $C_{E}=$ $\inf \{P \in \mathscr{P} ; P E=E\}$.) Then $Q_{1}$ and $Q_{2}$ are minimal in $\mathscr{P}$. It suffices to show that $Q_{1}=Q_{2}$. We argue by contradiction: Suppose $Q_{1} \neq Q_{2}$; then $Q_{1} Q_{2}=0$, by minimality. Let $\mathscr{P}_{c}$ denote the set of closed, central projections, i.e. $\mathscr{P}_{c}=\left\{I-P ; P \in \mathscr{P}_{0}\right\}$ and set $\mathscr{P} *=\mathscr{P}_{0} \cup \mathscr{P}_{c}$.

Claim. There is a $P \in \mathscr{P} *$ such that $Q_{1} \leqq P$ and $Q_{2} \leqq I-P$.

Assume, for a moment, this has been proved, and, for definiteness, let $P$ be open. Then there is an ideal $J$ in $\mathfrak{A}$ such that $J=\mathfrak{B P}$, and consequently there is an $A \in J$ with $A Q_{1} \neq 0$, since $0 \neq Q_{1} \leqq P$. On the other hand, $A Q_{2}=A P \cdot Q_{2}(I-P)=A Q_{2} P(I-P)=0$, contradicting our assumption that $\operatorname{ker} \pi_{1}=\operatorname{ker} \pi_{2}$, and we are through.

So it remains only to prove the Claim. Again we argue by contradiction: Assume there are distinct, minimal projections $Q_{1}$ and $Q_{2}$ in $\mathscr{P}$ such that,

(*) for all $P \in \mathscr{P} *,(I-P) Q_{1} \neq 0$ or $P Q_{2} \neq 0$.

Let $Q=Q_{1}+Q_{2}$ and consider the set:

$$
\mathscr{P}(Q)=\{P \in \mathscr{P} ; P Q=Q \text { or } P Q=0\} .
$$

By (*) and by minimality of $Q_{1}$ and $Q_{2}, \mathscr{P} * \subseteq \mathscr{P}(Q)$; and by minimality of $Q_{1}$ and $Q_{2}$ again, $\mathscr{P}(Q)$ is closed under finite unions, finite intersections and complementation. It follows that $\left\langle\mathscr{P}_{0}\right\rangle=\langle\mathscr{P} *\rangle \subseteq \mathscr{P}(Q)$. Now, by assumption there is a net $\left\{P_{\alpha}\right\}$ from $\left\langle\mathscr{P}_{0}\right\rangle$ such that $P_{\alpha} \rightarrow Q_{1}$ strongly, and, by minimality of $Q_{1}$, we may assume $P_{\alpha} \geqq Q_{1}$ for all $\alpha$. But then, since $\left\langle\mathscr{P}_{0}\right\rangle \subseteq \mathscr{P}(Q)$, also $P_{\alpha} \geqq Q_{1}+Q_{2}$ for all $\alpha$, and consequently $Q_{1}=$ $\lim P_{\alpha} \geqq Q_{1}+Q_{2}$, contradiction.

This completes the proof of the theorem.

3. REMARK. In the course of the proof we have also established the following: If $\mathfrak{A}$ is a $C^{*}$-algebra (separable or not) with the property that $\left\langle\mathscr{P}_{0}\right\rangle$ is dense in $\mathscr{P}$, then any two factor-representations of $\mathfrak{U}$ with the same kernel are quasi-equivalent.

\section{REFERENCES}

1. C. A. Akemann, The general Stone-Weierstrass problem, J. Functional Analysis 4 (1969), 277-294. MR 40 \#4772. 
2. J. Dixmier, Les $C^{*}$-algèbres et leurs représentations, Cahiers Scientifiques, fasc. 29, Gauthier-Villars, Paris, 1964. MR 30 \#1404.

3. —_ Les algèbres d'opérateurs dans l'espace Hilbertien, 2ième éd., GauthierVillars, Paris, 1969.

4. J. Glimm, Type I C*-algebras, Ann. of Math. (2) 73 (1961), 572-612. MR 23 \#A2066.

5. H. Halpern and T. Digernes, On open projections for $C^{*}$-algebras (to appear).

Department of Mathematics, University of California, los Angeles, CaliFORNIA 90024 